\title{
AN IMPROVED LOWER BOUND ON THE NUMBER OF LIMIT CYCLES BIFURCATING FROM A HAMILTONIAN PLANAR VECTOR FIELD OF DEGREE 7
}

\author{
TOMAS JOHNSON, WARWICK TUCKER
}

\begin{abstract}
The limit cycle bifurcations of a $\mathbf{Z}_{2}$ equivariant planar Hamiltonian vector field of degree 7 under $\mathbf{Z}_{2}$ equivariant degree 7 perturbation is studied. We prove that the given system can have at least 53 limit cycles. This is an improved lower bound for the weak formulation of Hilbert's 16th problem for degree 7 , i.e., on the possible number of limit cycles that can bifurcate from a degree 7 planar Hamiltonian system under degree 7 perturbation,
\end{abstract}

\section{INTRODUCTION}

Determining the number and location of (isolated) limit cycles for planar polynomial ordinary differential equations was posed as a grand challenge in Hilbert's seminal address to the International Congress of Mathematicians in 1900. Of the 23 problems presented by Hilbert, this (the 16th) turned out to be one of the most persistent: despite more than a century of intense research, not even the quadratic case has been resolved. For an overview of the progress that has been made to solve this problem we refer to (Ilyashenko 2002). Partial results for the quadratic case, and a general introduction to the bifurcation theory of planar polynomial vector fields can be found in (Roussarie 1998). What is known, is that any given polynomial vector field can have only a finite number of limit cycles; this is proved in (Écalle 1992, Ilyashenko 1991).

A restricted version of Hilbert's 16th problem introduced by Arnol'd, see e.g. (Arnold 1990), known as the weak or tangential Hilbert's 16th problem, asks for the number of limit cycles that can bifurcate from a perturbation of a Hamiltonian system, see e.g. (Christopher \& Li 2007). The weak Hilbert's 16th problem has been solved for the quadratic case, see (Chen et al. 2006).

In order to find Hamiltonian systems such that their perturbations have a maximum number of zeros, it is common to study symmetric Hamiltonians with a maximal number of centres, see e.g. (Li et al. 2002a, Li et al. 2002b, Zhou et al. 2007a, Zhou et al. 2007b). The specific perturbations are often constructed using the so-called detection function method, see (Li \& Huang 1987). In (Li \& Zhang 2004) a degree 7 perturbation of a $\mathbf{Z}_{8}$ equivariant system, with 49 limit cycles is constructed. As far as we know, this is the largest previously known lower bound on the number of limit cycles that can bifurcate through quintic perturbation of a quintic Hamiltonian vector field.

The aim of the present paper is to study a $\mathbf{Z}_{2}$ equivariant system with maximal number of centres, and prove that at least 53 limit cycles can bifurcate from it. We locate a suitable perturbation by conducting a similar study as in (Johnson \& Tucker 2009a, Johnson \& Tucker 2009b); this is described in detail in Section 3.2. We stress that our approach is completely rigorous, seeing that all numerics is done in interval arithmetic with directed rounding.

1.1. Abelian integrals. A classical method to prove the existence of limit cycles bifurcating from a continuous family of level curves of a Hamiltonian, $\gamma_{h} \subset H^{-1}(h)$, depending continuously on $h$, is to study Abelian integrals, or, more generally, the Melnikov function, see e.g. (Christopher \& Li 2007, Guckenheimer \& Holmes 1983). The closed level-curves of a polynomial Hamiltonian are called ovals. We denote the interior of an oval $D_{h}$, i.e. $\partial D_{h}=\gamma_{h}$. Given a Hamiltonian system and a perturbation,

$$
\left\{\begin{array}{l}
\dot{x}=-H_{y}(x, y)+\epsilon f(x, y) \\
\dot{y}=H_{x}(x, y)+\epsilon g(x, y)
\end{array}\right.
$$

2000 Mathematics Subject Classification. Primary: 37G15, Secondary: 37M20, 34C07, 65G20.

Key words and phrases. Abelian integrals, limit cycles, bifurcation theory, planar Hamiltonian systems, interval analysis. 
the Abelian integral, in general multi-valued, is defined as

$$
I(h)=\int_{\gamma_{h}} f(x, y) d y-g(x, y) d x .
$$

We denote the integrand $\omega$, and call it the 1-form associated with the perturbation. In this paper all perturbations are polynomial.

The most important property of Abelian integrals is described by the Poincaré-Pontryagin theorem.

Theorem 1.1 (Poincaré-Pontryagin). Let $P$ be the return map defined on some section transversal to the ovals of $H$, parametrised by the values $h$ of $H$, where $h$ is taken from some bounded interval (a,b). Let $d(h)=P(h)-h$ be the displacement function. Then, $d(h)=\epsilon(I(h)+\epsilon \phi(h, \epsilon)), \quad$ as $\quad \epsilon \rightarrow 0$, where $\phi(h, \epsilon)$ is analytic and uniformly bounded on a compact neighbourhood of $\epsilon=0, h \in(a, b)$.

Proof. see e.g. (Christopher \& Li 2007).

As a consequence of the above theorem, one can prove that a simple zero of $I(h)$ corresponds to a unique limit cycle bifurcating from the Hamiltonian system as $\epsilon \rightarrow 0$. In fact, to prove the existence of a limit cycle, it suffices to have a zero of odd order.

1.2. The Hamiltonian. We study Hamiltonian vector fields with a maximum number of centres. To generate symmetric such systems for vector fields of odd degree, $2 k+1$, one can study:

$$
\left\{\begin{array}{l}
\dot{x}=-y\left(y^{2}-1\right)\left(y^{2}-2\right) \cdots\left(y^{2}-k\right) \\
\dot{y}=x\left(x^{2}-1\right)\left(x^{2}-2\right) \cdots\left(x^{2}-k\right)
\end{array}\right.
$$

We study $\mathbf{Z}_{2}$ symmetric perturbations, and several of the monomial terms of the Abelian integral for such perturbations of (3) are equal. To construct perturbations with a maximum number of limit cycles it is desirable to break this symmetry. We therefore study the following system:

$$
\left\{\begin{array}{l}
\dot{x}=-y\left(y^{2}-1\right)\left(y^{2}-2\right)\left(y^{2}-3\right) \\
\dot{y}=x\left(x^{2}-1.1\right)\left(x^{2}-2.3\right)\left(x^{2}-3.6\right)
\end{array},\right.
$$

whose Hamiltonian function is given by:

$$
H(x, y)=\frac{x^{8}}{8}-\frac{7 x^{6}}{6}+\frac{1477 x^{4}}{400}-\frac{2277 x^{2}}{500}+\frac{y^{8}}{8}-y^{6}+\frac{11 y^{4}}{4}-3 y^{2} .
$$

The system has 49 equilibrium points and 42 periodic annuli, appearing in 14 classes, see Figure 1 . We label the classes of periodic annuli $\Gamma_{1}-\Gamma_{14}$, where the annuli $\Gamma_{1}-\Gamma_{8}$, are those with multiplicity 4 , labelled in decreasing order under inclusion, see Figure 2 and Table 1 . The annuli $\Gamma_{9}-\Gamma_{14}$ are labelled in increasing order under inclusion, see Figure 2 and Table 1.

\begin{tabular}{c|rrc} 
Periodic annulus & $h_{\min }$ & $h_{\max }$ & Expand/Contract \\
\hline 1 & -2.7626 & -1.9764 & expand \\
2 & -2.9112 & -2.7626 & expand \\
3 & -2.9764 & -2.7626 & expand \\
4 & -3.0362 & -2.9112 & expand \\
5 & -3.1014 & -2.9764 & expand \\
6 & -3.0362 & -2.9112 & expand \\
7 & -3.1014 & -2.9764 & expand \\
8 & -2.7626 & -2.6377 & contract \\
9 & -1.125 & 0 & contract \\
10 & -1.9112 & 1.6377 & contract \\
11 & -1.125 & -1 & contract \\
12 & -1.9676 & -1.9113 & expand \\
13 & -1.9112 & -1.125 & expand \\
14 & -1.125 & $\infty$ & expand
\end{tabular}

TABLE 1. The domains of the periodic annuli. The labels contract and expand refer to the behaviour of the ovals in an annulus as $h$ increases. 


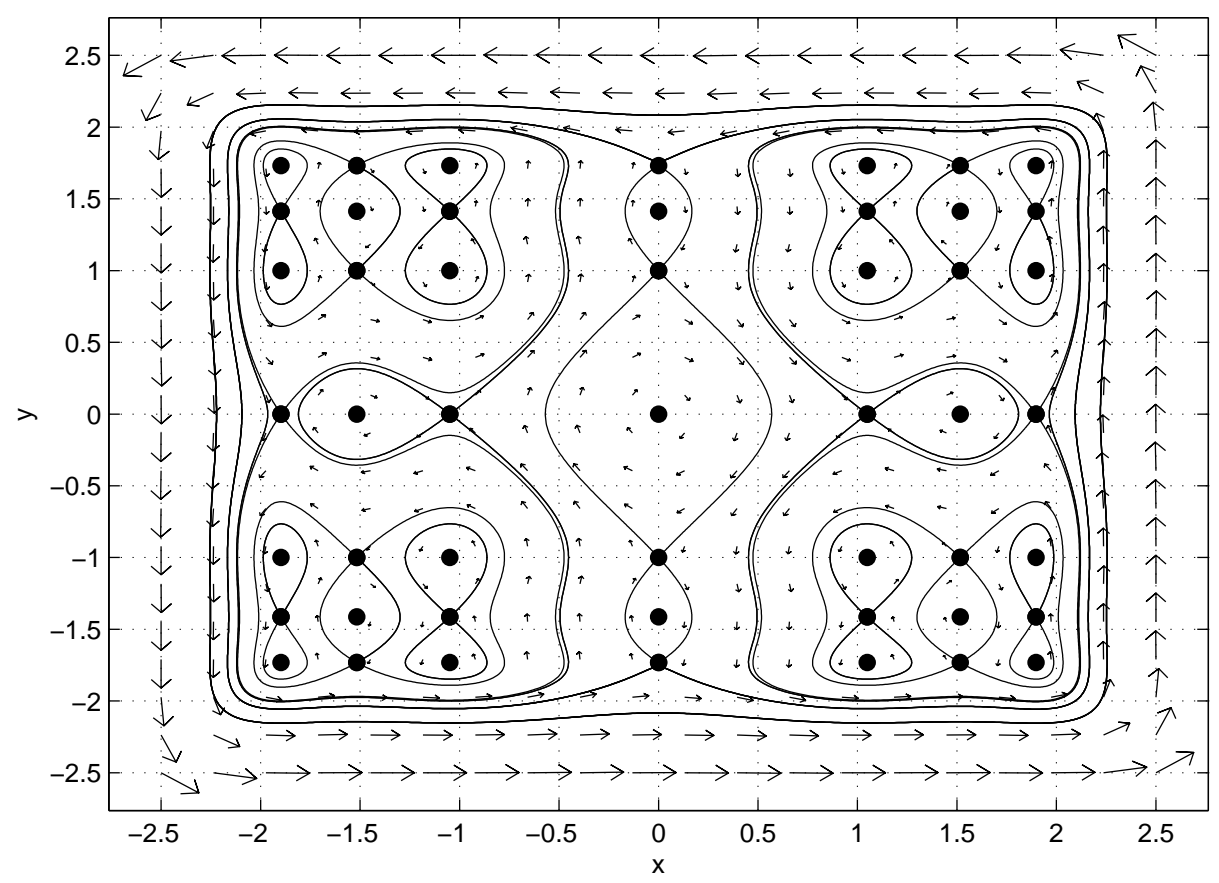

Figure 1. Phase portrait of the unperturbed Hamiltonian system.

(a)

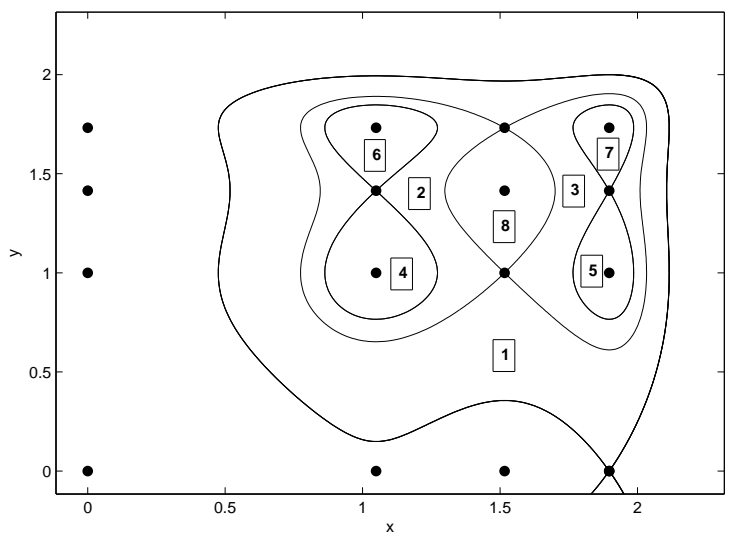

(b)

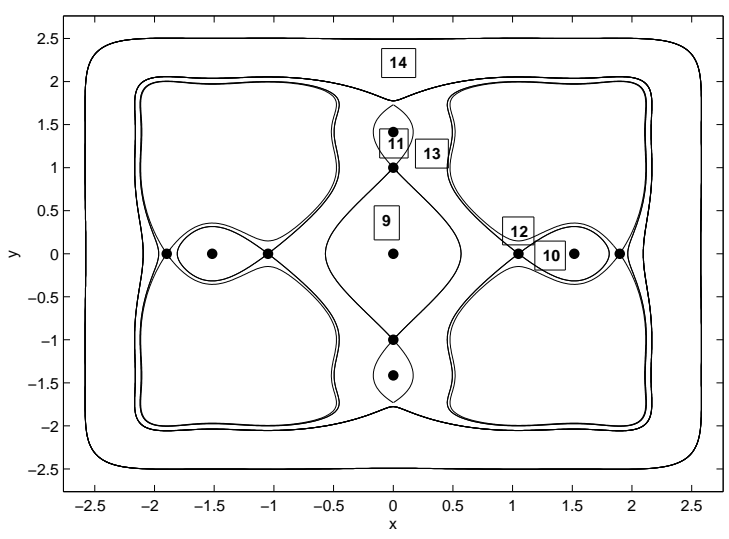

Figure 2. The periodic annuli, (a) $\Gamma_{1}-\Gamma_{8}$, and (b) $\Gamma_{9}-\Gamma_{14}$.

We are interested in limit cycles bifurcating from the periodic solutions of (4), corresponding to integral curves of (5).

We study the following $\mathbf{Z}_{2}$ equivariant perturbation of the Hamiltonian system (4), (6)

$\begin{array}{ccc}p(x, y) & := & \frac{\alpha_{00}}{2}+\frac{\alpha_{20}}{4} x^{2}+\frac{\alpha_{02}}{4} y^{2}+\frac{\alpha_{40}}{6} x^{4}+\frac{\alpha_{22}}{6} x^{2} y^{2}+\frac{\alpha_{04}}{6} y^{4}+\frac{\alpha_{60}}{8} x^{6}+\frac{\alpha_{42}}{8} x^{4} y^{2}+\frac{\alpha_{24}}{8} x^{2} y^{4}+\frac{\alpha_{06}}{8} y^{6} \\ f(x, y) & := & x p(x, y) \\ g(x, y) & := & y p(x, y)\end{array}$ 
Thus, the Abelian integral (2) reads,

$$
\begin{array}{rl}
I(h)=\int_{\gamma_{h}} & f d y-g d x=\int_{\gamma_{h}} x p d y-y p d x=\int_{D_{h}}\left(2 p+x \frac{\partial p}{\partial x}+y \frac{\partial p}{\partial y}\right) d x \wedge d y \\
=\int_{D_{h}} & \left(\alpha_{00}+\alpha_{20} x^{2}+\alpha_{02} y^{2}+\alpha_{40} x^{4}+\alpha_{22} x^{2} y^{2}+\alpha_{04} y^{4}\right. \\
& \left.+\alpha_{60} x^{6}+\alpha_{42} x^{4} y^{2}+\alpha_{24} x^{2} y^{4}+\alpha_{06} y^{6}\right) d x \wedge d y
\end{array}
$$

\section{Results}

Theorem 2.1. Consider the Hamiltonian vector field (4), perturbed as in (6). Then one can choose $\alpha_{i j}$, such that, as $\epsilon \rightarrow 0$, at least 53 limit cycles appear in the configuration,

$$
\left(\Gamma_{2}^{3}\right)^{4}\left(\Gamma_{3}^{2}\right)^{4}\left(\Gamma_{4}\right)^{4}\left(\Gamma_{5}^{2}\right)^{4}\left(\Gamma_{6}^{3}\right)^{4}\left(\Gamma_{7}\right)^{4}\left(\Gamma_{8}\right)^{4}\left(\Gamma_{14}\right),
$$

see Figure 3.

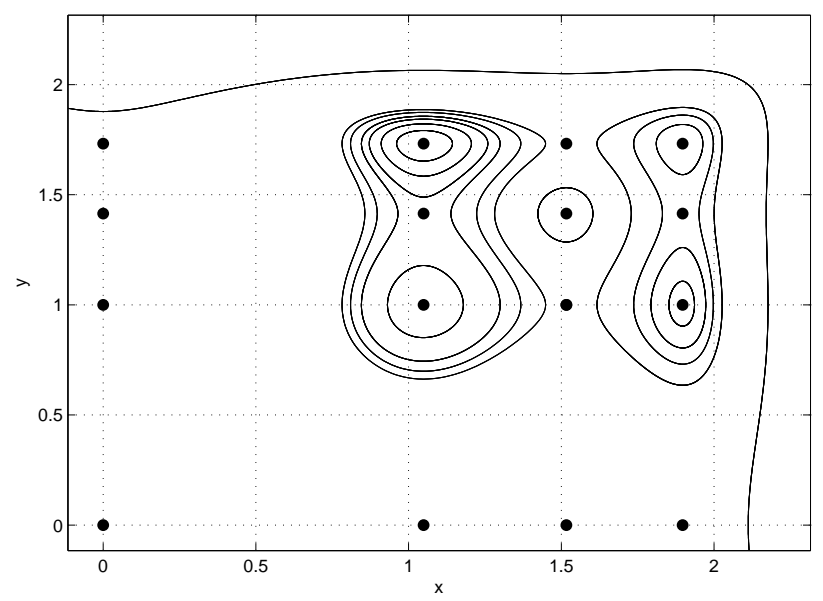

FiguRE 3. The ovals, from which the limit cycles bifurcate.

We use $Z(n+1, m)$ to denote the maximum number of limit cycles that can bifurcate from a Hamiltonian vector field of degree $n$, under a perturbation of order $m$. Obviously, $Z(n):=Z(n+1, n) \leq H(n)$, where $H(n)$ denotes the maximum number of limit cycles that a $n$th degree planar polynomial system can have. Some known results are $Z(2)=2$ (Chen et al. 2006), $Z(3) \geq 13$ (Li et al. 2009), $Z(4) \geq 15$ (Zhang et al. 2004), $Z(5) \geq 27$ (Johnson \& Tucker 2009b), Z(6) $\geq 35$ (Wang \& Yu 2005), Z(7) $\geq 49$ (Li \& Zhang 2004), $Z(9) \geq 80$ (Wang et al. 2006), and $Z(11) \geq 121$ (Wang \& Yu 2006).

Corollary 2.1. $Z(7) \geq 53$.

\section{Method}

3.1. Computer-aided proofs. Seeing that our proof relies upon a great deal of numerical computations, we have been very careful in validating the computational results. A numerical algorithm is said to be autovalidating if it produces a mathematically correct result, incorporating not only the discretisation errors of the numerical method, but also the computer's internal representation of the floating point numbers and its rounding procedures. The basic object in any such algorithm is an interval, whose endpoints are computer-representable floating points. All mathematical operations are performed in interval arithmetic with directed rounding to ensure the correctness of the result. For a thorough introduction to this topic we refer to (Moore 1966, Neumaier 1990). 
3.2. Computer-aided computation of Abelian integrals. We use the method developed in (Johnson \& Tucker 2008) to enclose the values of all Abelian integrals $I(h)$ appearing in our proof. This enables us to rigorously sample their values, i.e., for some discrete values of $h$, we can determine intervals such that $I(h) \in\left[I^{-}(h), I^{+}(h)\right]$. If we can find two ovals $\gamma_{h_{1}}$, and $\gamma_{h_{2}}$, such that all elements of $\left[I^{-}\left(h_{1}\right), I^{+}\left(h_{1}\right)\right]$ have the opposite sign as those of $\left[I^{-}\left(h_{2}\right), I^{+}\left(h_{2}\right)\right]$ then, by the intermediate value theorem, there exists $h^{*} \in\left(h_{1}, h_{2}\right)$, such that $I\left(h^{*}\right)=0$, and a neighbourhood of $\gamma_{h^{*}}$ that is either attracting or repelling for the perturbed vector field. Since $P_{\epsilon}$, the return map of the perturbed vector field, is analytic and non-constant, it has isolated fixed points. Thus, a zero of $I$ implies the existence of (at least) one limit cycle bifurcating from $\gamma_{h^{*}}$.

We recall that, in general, the Abelian integral is multi-valued, and the abovementioned computations are done for each continuous family of ovals separately. In the equivariant case at hand, $I(h)$ is identically the same on each of the different annuli within one annulus-class. Thus, one can trivially split the set of ovals corresponding to $H=h$ into natural subsets. This is crucial for the success of our approach, since for each limit cycle we find bifurcating from the annuli $\Gamma_{1}-\Gamma_{8}$ there will be three additional cycles that can be found by reflecting in the $x$ and $y$ axes.

In order to construct a perturbation such that the associated Abelian integral has a given number of zeros, the perturbation has to be chosen in a careful manner. We use the same heuristic procedure as in (Johnson \& Tucker 2009a, Johnson \& Tucker 2009b) to generate the coefficients of a suitable candidate form $\omega$. The first part of our approach is to integrate monomial forms at some points, $h_{1}, \ldots, h_{N}$, and then to specify the coefficients of

(7) $d \omega=\left(\alpha_{00}+\alpha_{20} x^{2}+\alpha_{02} y^{2}+\alpha_{40} x^{4}+\alpha_{22} x^{2} y^{2}+\alpha_{04} y^{4}+\alpha_{60} x^{6}+\alpha_{42} x^{4} y^{2}+\alpha_{24} x^{2} y^{4}+\alpha_{06} y^{6}\right) d x \wedge d y$,

such that the Abelian integrals vanish:

$$
I\left(h_{\ell}\right)=\int_{\gamma_{h_{\ell}}} \omega=0, \quad \ell=1, \ldots, N
$$

Therefore, let

$$
I_{i j}(h)=\int_{D_{h}} x^{i} y^{j} d x \wedge d y
$$

where $\partial D_{h}=\gamma_{h}$. Then we have the following linear decomposition

$$
\begin{aligned}
I(h) & =\alpha_{00} I_{00}(h)+\alpha_{20} I_{20}(h)+\alpha_{02} I_{02}(h)+\alpha_{40} I_{40}(h)+\alpha_{22} I_{22}(h) \\
& +\alpha_{04} I_{04}(h)+\alpha_{60} I_{60}(h)+\alpha_{42} I_{42}(h)+\alpha_{24} I_{24}(h)+\alpha_{06} I_{06}(h) .
\end{aligned}
$$

Note, this method can automatically give any configuration of limit cycles generated by 9 zeros, i.e., up to 36 limit cycles. To find a better set of candidate coefficients we note that the system has the property that the Abelian integral (10) is multi-valued for some pieces of the domain, see Table 1. This property indicates that it should be possible, as in (Johnson \& Tucker 2009a), to force those I's that have joint domains to oscillate together.

Given some candidate coefficients of the form $\omega$, we calculate the $I_{i j}(h)$ at intermediate ovals, $\tilde{h}_{1}<h_{1}<$ $\tilde{h}_{2}<\cdots<h_{N}<\tilde{h}_{N+1}$. If the linear combination (10) of the $I_{i j}(\tilde{h})$ has validated sign changes between the sample points we are done: it has been proved that the corresponding perturbation yields bifurcations with at least the given number of limit cycles as $\epsilon \rightarrow 0$.

3.3. Computational results. Using the method described above to generate candidate coefficients for $\omega$, we get the result listed in Table 2 .

The next step is to validate that the generated coefficients yield the expected behaviour. Therefore, we enclose the value of the corresponding Abelian integrals at intermediate ovals. As is shown in Table 3, the generated coefficients correspond to a perturbation for which the claimed number of limit cycles bifurcate from the given Hamiltonian as $\epsilon \rightarrow 0$. The graphs of the Abelian integrals for $\Gamma_{2}, \Gamma_{3}, \Gamma_{4}, \Gamma_{5}, \Gamma_{6}, \Gamma_{7}, \Gamma_{8}$, and $\Gamma_{14}$, from which it bifurcates limit cycles, are shown in Figure 4.

All computations were performed on a Quad-Core AMD Opteron Processor 8354 2.2GHz, 64bit processor with $32 \mathrm{~Gb}$ of RAM. The program was compiled with gcc, version 4.1.2. The software for interval arithmetic was provided by the CXS-C package, version 2.2.3, see (CXSC 2008, Hammer et al. 1995). The total run-time 

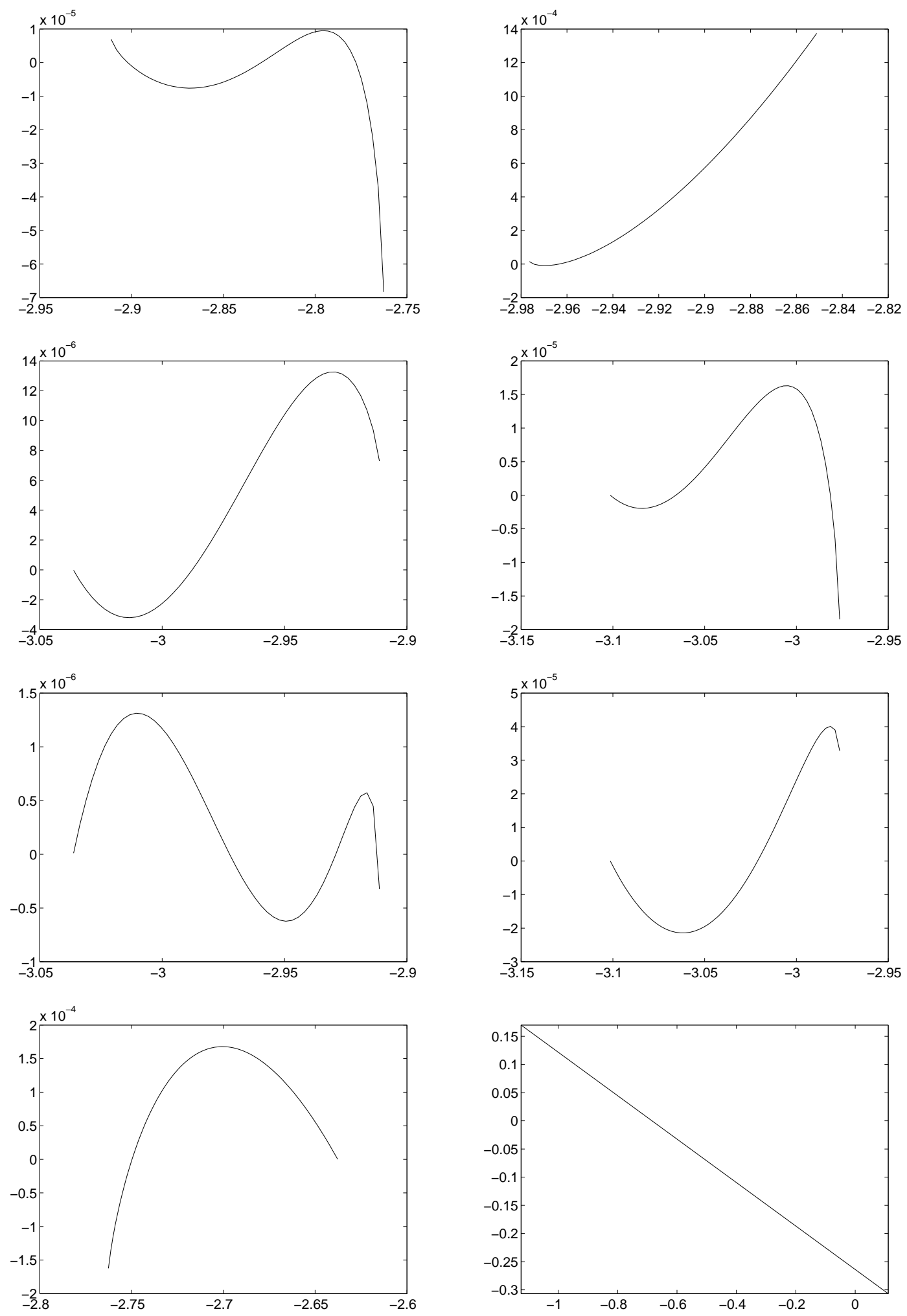

FIguRE 4 . The graphs of the Abelian integrals on $\Gamma_{2}-\Gamma_{8}$ and $\Gamma_{14}$. 


\begin{tabular}{l|r}
$\alpha_{00}$ & 0.30961876 \\
$\alpha_{20}$ & 1.0000000 \\
$\alpha_{02}$ & -1.7072698 \\
$\alpha_{40}$ & -0.59879205 \\
$\alpha_{22}$ & 0.25041556 \\
$\alpha_{04}$ & 0.81262742 \\
$\alpha_{60}$ & 0.10095772 \\
$\alpha_{42}$ & -0.057802570 \\
$\alpha_{24}$ & 0.0051675287 \\
$\alpha_{06}$ & -0.13625345
\end{tabular}

TABLE 2. The generated coefficients of the perturbation (6).

\begin{tabular}{cc|c} 
Periodic annulus & $h$ & $I(h)$ \\
\hline 2 & -2.9110 & {$[5.7976,7.4677] \times 10^{-6}$} \\
2 & -2.8690 & {$[-8.5663,-6.6829] \times 10^{-6}$} \\
2 & -2.7956 & {$[0.8383,1.0598] \times 10^{-5}$} \\
2 & -2.7630 & {$[-6.1764,-5.9324] \times 10^{-5}$} \\
3 & -2.9764 & {$[1.2772,1.5956] \times 10^{-5}$} \\
3 & -2.9680 & {$[-1.0051,-6.7627] \times 10^{-5}$} \\
3 & -2.8000 & {$[2.4572,2.4619] \times 10^{-3}$} \\
4 & -3.0130 & {$[-3.2949,-3.1166] \times 10^{-6}$} \\
4 & -2.9290 & {$[1.3055,1.3467] \times 10^{-5}$} \\
5 & -3.0840 & {$[-2.2207,-1.6995] \times 10^{-6}$} \\
5 & -3.0040 & {$[1.5658,1.6910] \times 10^{-5}$} \\
5 & -2.7964 & {$[-2.4966,-2.4959] \times 10^{-3}$} \\
6 & -3.0110 & {$[1.2661,1.3593] \times 10^{-6}$} \\
6 & -2.9490 & {$[-7.0838,-5.3883] \times 10^{-7}$} \\
6 & -2.9163 & {$[4.7068,6.6876] \times 10^{-7}$} \\
6 & -2.9113 & {$[-2.8479,-0.8376] \times 10^{-7}$} \\
7 & -3.0630 & {$[-2.1895,-2.0970] \times 10^{-5}$} \\
7 & -2.9820 & {$[3.9247,4.0927] \times 10^{-5}$} \\
8 & -2.6990 & {$[1.6729,1.6816] \times 10^{-4}$} \\
8 & -2.7620 & {$[-1.4908,-1.4782] \times 10^{-4}$} \\
14 & -1.1240 & {$[0.1638,0.1726]$} \\
14 & -0.5000 & {$[-0.1311,-0.1225]$}
\end{tabular}

TABLE 3. The computed enclosures of the Abelian integrals.

of the validated program (Johnson \& Tucker 2008), to calculate the 22 Abelian integrals necessary for the proof, was 33.57 hours.

\section{Some CONCLUding REMARKS}

We have continued the study of lower bounds for the weak Hilbert 16th problem for odd degrees from (Johnson \& Tucker 2009a), where we obtained a new lower bound for the degree five case, and studied the degree seven case. This is probably, however, the limit of our heuristic approach based on $\mathbf{Z}_{2}$ symmetric perturbations. The reason is that even though our procedure is mostly automated it still requires human input, and the next case, degree 9, would have 15 parameters to be controlled by hand. A future project is therefore to develop an automated method to pick parameters for $\mathbf{Z}_{2}$ symmetric perturbations of the symmetric centres of a Hamiltonian vector field of odd degree and with maximal number of centres.

For vector fields of even order no such simple symmetries as the restriction to the study of even Hamiltonians and perturbations exist. Our method is therefore not directly applicable in this situation. We believe, 
TOMAS JOHNSON, WARWICK TUCKER

however, that it should be possible to improve the bound for the degree four case using our integrator (Johnson \& Tucker 2008).

\section{REFERENCES}

V.I. Arnol'd [1990] "Ten problems. Theory of singularities and its applications" Adv. Soviet Math. 1, 1-8.

Chen, F., Li, C., Llibre, J.,Zhang, Z. [2006] "A unified proof on the weak Hilbert 16th problem for $n=2 . " J$. Differential Equations 221, 309-342.

Christopher, C. \& Li, C. [2007] Limit cycles of differential equations. (Advanced Courses in Mathematics. CRM Barcelona. Birkhäuser Verlag, Basel.)

CXSC [2008]. C++ eXtension for Scientific Computation, version 2.2.3. (Available from http://www.math.uniwuppertal.de/org/WRST/xsc/cxsc.html)

Ecalle, J. [1992] Introduction aux fonctions analysables et preuve constructive de la conjecture de Dulac. (French) Introduction to analyzable functions and constructive proof of the Dulac conjecture (Actualités Mathématiques. Current Mathematical Topics Hermann, Paris.)

Guckenheimer, J. \& Holmes, P., [1983] Nonlinear Oscillations, Dynamical Systems, and Bifurcations of Vector Fields (Applied Mathematical Sciences, 42. Springer-Verlag, New York)

Hammer, R., Hocks, M., Kulisch, U., \& Ratz, D. [1995] C++ Toolbox for Verified Computing (Springer-Verlag, New York)

Horozov, E. \& Iliev, I.D. [1998] "Linear estimate for the number of zeros of Abelian integrals with cubic Hamiltonians." Nonlinearity 11, 1521-1537.

Il'yashenko, Yu.S. [1991] Finiteness theorems for limit cycles. (Translations of Mathematical Monographs, 94. American Mathematical Society, Providence, RI.)

Il'yashenko, Yu.S. [2002] "Centennial history of Hilbert's 16th problem" Bull. Amer. Math. Soc. (N.S.) 39, 301-354

Johnson, T. \& Tucker, W. [2008] "On a computer-aided approach to the computation of Abelian integrals", submitted.

Johnson, T. \& Tucker, W. [2009] "A rigorous study of possible configurations of limit cycles bifurcating from a hyper-elliptic Hamiltonian of degree five", Dynamical Systems - An international journal 24, 237-247.

Johnson, T. \& Tucker, W. [2009] "An improved lower bound on the number of limit cycles bifurcating from a quintic Hamiltonian planar vector field under quintic perturbation", Internat. J. Bifur. Chaos Appl. Sci. Engrg., to appear.

Li, J., Chan, H.S.Y., Chung, K. W. [2002] "Bifurcations of limit cycles in a $Z_{6}$-equivariant planar vector field of degree 5." Sci. China Ser. A 45, 817-826.

Li, J., Chan, H.S.Y., Chung, K. W. [2002] "Some lower bounds for $H(n)$ in Hilbert's 16th problem." Qual. Theory Dyn. Syst. 3 (2002), no. 2, 345-360.

Li, J.B. \& Huang, G.M., [1987] "Bifurcations of limit cycles forming compound eyes in the cubic system." Chinese Ann. Math. Ser. B 8, 391-403.

Li, J., Liu, C., Jiazhong, Y., [2009] "A cubic system with thirteen limit cycles." J. Differential Equations 246, 3609-3619.

Li, J. \& Zhang, M., [2004] "Bifurcations of limit cycles in a $Z_{8}$-equivariant planar vector field of degree 7." J. Dynam. Differential Equations $16,1123-1139$.

Moore, R.E. [1966] Interval Analysis, (Prentice-Hall, Englewood Cliffs, New Jersey.)

Neumaier, A. [1990] Interval Methods for Systems of Equations. (Encyclopedia of Mathematics and its Applications 37, Cambridge Univ. Press, Cambridge.)

Roussarie, R. [1998] Bifurcation of planar vector fields and Hilbert's sixteenth problem (Progress in Mathematics, 164. Birkhäuser Verlag, Basel.)

Wang, S. \& Yu, P. [2005] "Bifurcation of limit cycles in a quintic Hamiltonian system under a sixth-order perturbation." Chaos Solitons Fractals 26, 1317-1335.

Wang \& S., Yu, P., [2006] "Existence of 121 limit cycles in a perturbed planar polynomial Hamiltonian vector field of degree 11." Chaos Solitons Fractals 30, 606-621.

Wang, S., Yu, P., Li, J. [2006] "Bifurcation of limit cycles in $Z_{10}$-equivariant vector fields of degree 9." Internat. J. Bifur. Chaos Appl. Sci. Engrg. 16, 2309-2324.

Zhou, H., Xu, W., Li, S., Zhang, Y. [2007] "On the number of limit cycles of a cubic polynomials Hamiltonian system under quintic perturbation." Appl. Math. Comput. 190, 490-499.

Zhou, H., Xu, W., Zhao, X., Li, S. [2007] "Detection function method and its application to a class of quintic Hamiltonian systems with quintic perturbations." Appl. Math. Comput. 191, 490-503.

Zhang, T., Han, M., Zang, H., Meng, X. [2004] "Bifurcations of limit cycles for a cubic Hamiltonian system under quartic perturbations." Chaos Solitons Fractals 22, 1127-1138.

Department of Mathematics, Uppsala University, Box 480, 75106 Uppsala, Sweden

E-mail address: tomas.johnson@math.uu.se, warwick.tucker@math.uu.se 